\title{
Variations in Practice among Asia-Pacific Surgeons and Recommendations for Managing Cervical Myelopathy: The First Asia-Pacific Spine Society Collaborative Study
}

\author{
Jason Pui Yin Cheung ${ }^{1}$, Prudence Wing Hang Cheung ${ }^{1}$, Chee Kidd Chiu ${ }^{2}$, \\ Chris Yin Wei Chan ${ }^{2}$, Mun Keong Kwan ${ }^{2}$ \\ ${ }^{1}$ Department of Orthopaedics and Traumatology, The University of Hong Kong, Hong Kong, SAR, China \\ ${ }^{2}$ Department of Orthopaedic Surgery, National Orthopaedic Centre of Excellence for Research and Learning, Faculty of Medicine, University of Malaya, \\ Kuala Lumpur, Malaysia
}

Study Design: Surgeon survey.

Purpose: To study the various surgical practices of different surgeons in the Asia-Pacific region.

Overview of Literature: Given the diversity among Asia-Pacific surgeons, there is no clear consensus on the preferred management strategies for cervical myelopathy. In particular, the role of prophylactic decompression for silent cervical spinal stenosis is under constant debate and should be addressed.

Methods: Surgeons from the Asia-Pacific Spine Society participated in an online questionnaire comprising 50 questions. Data on clinical diagnosis, investigations and outcome measures, approach to asymptomatic and silent cervical spinal stenosis, guidelines for surgical approach, and postoperative immobilization were recorded. All parameters were analyzed by the Mantel-Haenszel test.

Results: A total of 79 surgeons from 16 countries participated. Most surgeons used gait disturbance $(60.5 \%)$ and dyskinetic hand movement (46.1\%) for diagnosis. Up to $5.2 \%$ of surgeons would operate on asymptomatic spinal stenosis, and $18.2 \%$ would operate on silent spinal stenosis. Among those who would not operate, most (57.1\%) advised patients on avoidance behavior and up to $9.5 \%$ prescribed neck collars. For ossification of the posterior longitudinal ligament (OPLL), anterior removal was most commonly performed for one-level disease $(p<0.001)$, whereas laminoplasty was most commonly performed for two- to four-level disease $(p=0.036)$. More surgeons considered laminectomy and fusion for multilevel OPLL. Most surgeons generally preferred to use a rigid neck collar for 6 weeks postoperatively $(p<0.001)$.

Conclusions: The pooled recommendations include prophylactic or early decompression surgery for patients with silent cervical spinal stenosis, particularly OPLL. Anterior decompression is primarily suggested for one- or two-level disease, whereas laminoplasty is preferred for multilevel disease.

Keywords: Cervical myelopathy; Asia-Pacific; Ossification of the posterior longitudinal ligament; Silent; Cervical stenosis

Received Jun 12, 2018; Revised Jun 28, 2018; Accepted Jul 3, 2018

Corresponding author: Jason Pui Yin Cheung

Department of Orthopaedics and Traumatology, The University of Hong Kong, 5th Floor Professorial Block, 102 Pokfulam Road, Pokfulam, Hong Kong, SAR, China

Tel: +852-2255-4581, Fax: +852-2817-4392, E-mail: cheungjp@hku.hk 


\section{Introduction}

With an aging population, the number of patients presenting with cervical myelopathy is increasing worldwide. This disorder is characterized by upper and lower limb numbness and weakness, with difficulties in ambulation and sphincter control. Further, it is the leading cause of spinal cord dysfunction [1]. Loss of the ability to transfer loads through intervertebral discs and spinal segment hypermobility causes osteophyte formation and buckling of the ligamentum flavum as a result of loss of disc height. In Asia, ossification of the posterior longitudinal ligament (OPLL) is also an important and common cause of cervical myelopathy. The prevalence in the general Japanese population is reported as $1.9 \%-4.3 \%$ and up to $25 \%$ for patients with cervical myelopathy [2]. An international study reported that Asia-Pacific centers manage more cases of OPLL (35\%) than North American centers (12\%) [3].

Surgery is typically recommended for these patients, as most display neurological deterioration over time. Anterior, posterior, or combined approaches may adequately decompress the spinal canal. The choice of approach is variable and generally depends on factors such as cause of compression, number of affected segments, and the surgeon's experience. Anterior approaches are excellent for disc herniations and bone spurs but are less extensile than posterior approaches. However, posterior approaches usually permit only indirect decompression and may lead to further axial neck pain. In addition to the lack of consensus on surgical approaches, there is also a lack of consensus on preoperative assessment methods, imaging [4], and the need for postoperative immobilization [5]. Prophylactic decompression for silent cervical canal stenosis is performed by some surgeons but remains controversial [6].

The lack of definitive evidence for controversial areas of spine surgery and the variable distribution of pathology in different geographic regions lead to different practice patterns. Many spinal conditions are managed very differently depending on the region and the experience of the surgeon [7-9]. Cultural, ethnic, and socioeconomic factors may come into play to determine the treatment regimen. Given the diversity in the Asia-Pacific region, the mixed experiences and practice guidelines will provide an intriguing outlook of managing both spondylotic and OPLL causes of cervical myelopathy. Hence, this study aims to identify any consensus and to develop recommendations from Asia-Pacific surgeons.

\section{Materials and Methods}

\section{Subjects}

Surgeons from the Asia-Pacific Spine Society (APSS) participated in an electronic survey exploring aspects of perioperative management of cervical myelopathy. Members of the APSS include surgeons from Australia, Bangladesh, China, Hong Kong, India, Indonesia, Japan, Korea, Malaysia, Myanmar, Nepal, Pakistan, Philippines, Singapore, Sri Lanka, Thailand, Taiwan, Turkey, and Vietnam. The questionnaire was distributed via REDCap (Vanderbilt, Nashville, TN, USA). All surgeons who were members of the APSS were invited to participate. The questionnaire was circulated during the period from January to April 2017.

\section{Questionnaire}

The online questionnaire contained 50 questions grouped into five sections based on the participant's background details, preoperative assessment principles (clinical diagnosis of cervical myelopathy, imaging methods, and outcome scores used), approach to asymptomatic and silent cervical spinal stenosis, surgical approach to non-OPLL and OPLL cases, and postoperative immobilization practice. The responses were based on 5-point Likert scales ('least important,' 'less important,' 'neutral,' 'more important,' or 'most important' for clinical and radiological parameters; and 'most favorable,' 'more favorable, 'neutral', 'less favorable,' or 'least favorable' for surgical techniques).

\section{1) Background details}

The participants provided information regarding their country of origin, years of experience practicing spine surgery, number of cases operated on per year, whether they managed cervical myelopathy on a daily basis, familiarity with anterior or posterior approaches or with both approaches, and whether they managed occipital-cervical junction pathologies.

\section{2) Preoperative assessment}

The participants reported whether they considered various clinical findings important for the diagnosis of cervical myelopathy. The clinical parameters included bilateral upper and lower limb numbness and weakness, gait disturbance, dyskinetic hand movement, hyperre- 
flexia, and sphincter dysfunction. Clinical examination tests included neck range of motion, Spurling's sign, Lhermitte's sign, limb power, limb sensation, presence of brisk reflexes, finger escape sign, 10-second test, observed dyskinetic movements, Hoffman's sign, reverse supinator reflex, scapulohumeral reflex, upgoing Babinski response, clonus, unsteady gait, cerebellar signs, rectal examination, and functional testing (writing or buttoning). Methods of investigation were also assessed, including cervical spine radiographs (anteroposterior and lateral), dynamic flexion-extension lateral cervical spine radiographs, open mouth view, computed tomography (CT), magnetic resonance imaging (MRI), nerve conduction study, and electromyography. The outcome scores utilized, including the Nurick scale, Japanese Orthopaedic Association (JOA) score, and Neck Disability Index (NDI), were also reported.

\section{3) Approach to cervical spinal stenosis}

The participants were asked whether they operated on asymptomatic (only stenosis on imaging without any myelopathy symptoms) and/or silent (no significant functional deficit, JOA score >15) cervical spinal stenosis, and how they decided when to intervene. In addition, for participants who did not operate on these patients, how they advised patients regarding activities and risks was also investigated.

\section{4) Surgical approach}

The participants reported their approach to one-level, two-level, three-level, and four-level diseases of nonOPLL and OPLL origin. Anterior approaches included anterior discectomy and fusion (ACDF), corpectomy, and disc replacement. Posterior approaches included laminectomy, laminectomy and fusion, laminoplasty, and laminoplasty and fusion. The participants were also given an additional option of a combined approach. For ACDF or corpectomy, the participants reported whether they used bone graft or cage only or with plating. For posterior fusion, the participants reported whether they used lateral mass screws or pedicle screws. For laminoplasty, the participants reported whether they used the single-door technique with plating or suture fixation, or the doubledoor technique. For atlantoaxial surgery, the participants reported whether they used transarticular screws, C1 lateral mass screws, C2 pedicle screws, or laminar screws, or wiring techniques.

\section{5) Postoperative management}

The participants reported whether they used postoperative immobilization methods, including the soft or rigid neck collar and the Halo immobilization device, or allowed free mobilization for the following operations: ACDF, ACDF with plating, corpectomy, corpectomy with plating, laminectomy with fusion, laminectomy alone, laminoplasty, atlantoaxial fusion, and occipital-cervical fusion. The duration of immobilization was also reported.

\section{Analysis}

After all the questionnaires had been completed, the results and analyses were discussed among the investigators of the study to provide recommendations based on the data. Statistical analysis was performed by IBM SPSS ver. 23.0 (IBM Corp., Armonk, NY, USA). Basic demographics and relationships between study parameters were investigated by the chi-square test of independence. Fisher's exact test was used for parameter values less than 5. After the relationships between the level of disease and the surgical approach for OPLL and non-OPLL cases had been established, the Mantel-Haenszel test was used to assess the relationships between the surgeon's experience (in years) and the surgical approach, and management of silent and asymptomatic cervical spinal stenosis, while controlling for the level of disease. Statistical significance was set at $p<0.05$.

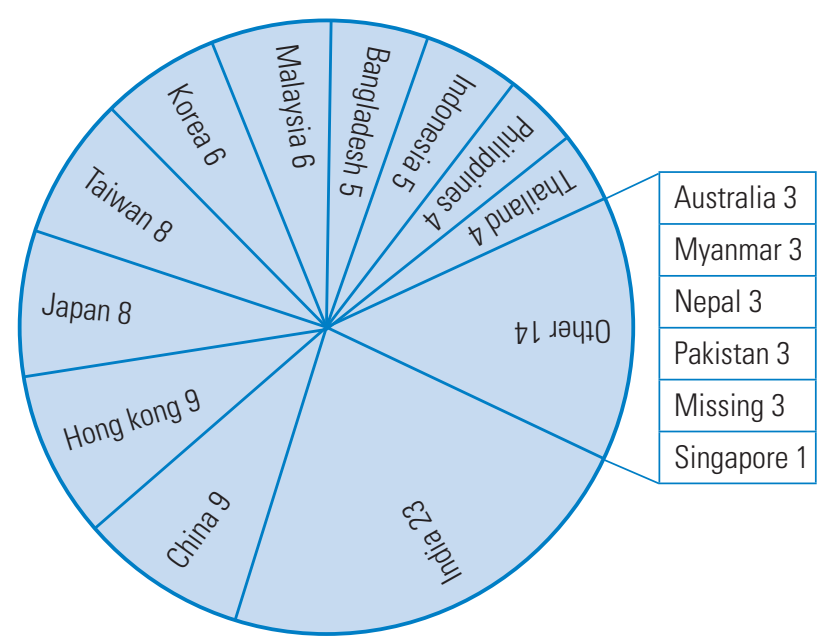

Fig. 1. Frequency of participation of members of the Asia-Pacific Spine Society (unit: \%). 


\section{Results}

Surgeons from all countries in the APSS were represented $(\mathrm{n}=16)$ except Indonesia, Turkey, and Vietnam (Fig. 1). Among the participants, there was a good spread of surgical experience: $47.0 \%$ had practiced for $\leq 10$ years, $24.1 \%$ had practiced for 11 to 20 years, and $22.9 \%$ had practiced for $>20$ years. Most surgeons (84.2\%) operated on fewer than 40 cervical myelopathy cases a year, $9.2 \%$ operated on 41 to 60 cases a year, $2.6 \%$ operated on 61 to 80 cases a year, and $2.6 \%$ operated on 81 to 100 cases a year. Participants who were more experienced were more likely to operate on the occipital-cervical junction $(\leq 10$ years experience, $47.4 \%$; 11 to 20 years experience, $80.0 \%$; >20 years experience, $78.9 \%$; $p=0.014)$.

Most surgeons (60.5\%) felt that gait disturbance was 'most important' for the diagnosis of cervical myelopathy, followed by dyskinetic hand movement (46.1\%), hyperreflexia (44.7\%), and sphincter dysfunction (35.1\%). None of the surgeons believed that bilateral upper and lower limb numbness was 'most important.' Only 35\% to $45 \%$ felt these symptoms to be 'more important' than other symptoms. Hoffman's sign (64.9\%), unsteady gait (63.6\%), and brisk reflexes (49.4\%) were the three 'most important' clinical signs for diagnosis. All surgeons would obtain an MRI in their preoperative investigation, but only $81.8 \%$ would obtain a lateral cervical spine radiograph and $76.6 \%$ would obtain dynamic flexion-extension radiographs. Up to $41.6 \%$ of participants would perform CT preoperatively, and $14.3 \%$ would obtain nerve conduction studies routinely. Not all participants utilized objective outcome scores. Of those who did, most (83.1\%) utilized the JOA score, whereas $23.4 \%$ utilized the Nurick scale and $22.1 \%$ utilized the NDI.

Only $5.2 \%$ of participants would operate on asymptomatic spinal stenosis, and $18.2 \%$ would operate on silent spinal stenosis. Most of those who would perform surgery for asymptomatic stenosis were from Thailand (33.3\%), China (28.6\%), and Korea (20.0\%). The decision for surgery was based on the patient's decision after the MRI findings were explained to the patient. Among those who would operate on silent stenosis, most were from Hong Kong (71.4\%), Japan (16.7\%), and India (16.7\%). The decision for surgery was based on the patient's decision after the MRI findings were explained and also on whether the patient reported functional difficulties. Among those who would not operate on silent stenosis, most (57.1\%)
Table 1. Surgical approach for non-ossification of posterior longitudinal ligament causes of cervical myelopathy

\begin{tabular}{|c|c|c|c|}
\hline \multirow{2}{*}{ Levels of disease } & \multicolumn{2}{|c|}{ Surgical approach } & \multirow{2}{*}{$p$-value } \\
\hline & Yes & No & \\
\hline Anterior discectomy and fusion & & & $<0.001^{*}$ \\
\hline 1 & 57 & 22 & \\
\hline 2 & 32 & 47 & \\
\hline 3 & 13 & 66 & \\
\hline 4 & 4 & 75 & \\
\hline Anterior corpectomy & & & $0.001^{*}$ \\
\hline 1 & 1 & 78 & \\
\hline 2 & 16 & 63 & \\
\hline 3 & 3 & 76 & \\
\hline 4 & 0 & 79 & \\
\hline Posterior laminectomy & & & 0.762 \\
\hline 1 & 3 & 76 & \\
\hline 2 & 4 & 75 & \\
\hline 3 & 6 & 73 & \\
\hline 4 & 5 & 74 & \\
\hline Posterior laminectomy and fusion & & & $0.001^{*}$ \\
\hline 1 & 0 & 79 & \\
\hline 2 & 3 & 76 & \\
\hline 3 & 7 & 72 & \\
\hline 4 & 11 & 68 & \\
\hline Posterior laminoplasty & & & $<0.001^{*}$ \\
\hline 1 & 0 & 79 & \\
\hline 2 & 4 & 75 & \\
\hline 3 & 23 & 56 & \\
\hline 4 & 30 & 49 & \\
\hline Posterior laminoplasty and fusion & & & $0.001^{*}$ \\
\hline 1 & 1 & 78 & \\
\hline 2 & 0 & 79 & \\
\hline 3 & 7 & 72 & \\
\hline 4 & 5 & 74 & \\
\hline
\end{tabular}

${ }^{*} p<0.05$ (statistical significance).

advised avoiding extreme activities and sports, and up to 9.5\% prescribed neck collars. There was no relationship between the decision to operate and the surgeon's experience for asymptomatic $(p=0.515)$ or silent $(p=0.521)$ stenosis.

Most surgeons (77.9\%) performed both anterior and posterior surgery without a definite preference for either ( $p=0.383)$. The overall practice differed between non- 


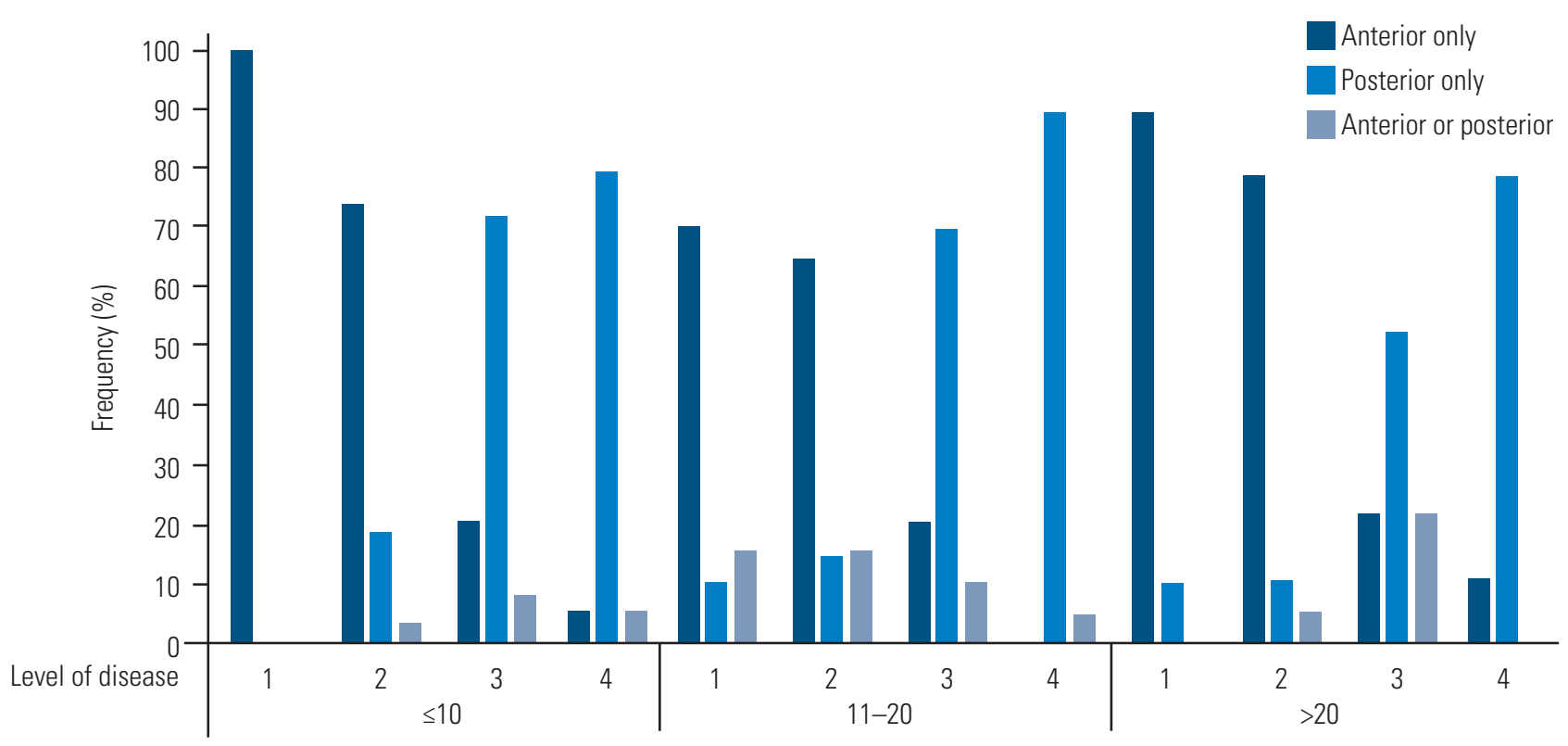

Spine surgery experience (yr)

Fig. 2. Years of surgeon's experience in relation to choice of surgical approach for cervical myelopathy not caused by ossification of the posterior longitudinal ligament.

Table 2. Surgical approach for non-ossification of posterior longitudinal ligament causes of cervical myelopathy with reference to surgical experience

\begin{tabular}{|c|c|c|c|}
\hline Years of practicing spine surgery & Levels of disease & Surgical approach & $p$-value \\
\hline \multirow[t]{6}{*}{$\leq 10$} & 1 to 4 levels & ACDF & 0.055 \\
\hline & & Anterior corpectomy & 0.855 \\
\hline & & Posterior laminectomy & 0.990 \\
\hline & & Posterior laminectomy and fusion & 0.299 \\
\hline & & Posterior laminoplasty & 0.347 \\
\hline & & Laminoplasty and fusion & 0.782 \\
\hline \multirow[t]{6}{*}{$11-20$} & 1 to 4 levels & ACDF & $0.013^{*}$ \\
\hline & & Anterior corpectomy & 0.598 \\
\hline & & Posterior laminectomy & 0.857 \\
\hline & & Posterior laminectomy and fusion & 0.304 \\
\hline & & Posterior laminoplasty & 0.168 \\
\hline & & Laminoplasty and fusion & 0.955 \\
\hline \multirow[t]{6}{*}{$>20$} & 1 to 4 levels & ACDF & 0.912 \\
\hline & & Anterior corpectomy & 0.558 \\
\hline & & Posterior laminectomy & 0.938 \\
\hline & & Posterior laminectomy and fusion & 0.939 \\
\hline & & Posterior laminoplasty & 0.900 \\
\hline & & Laminoplasty and fusion & 0.954 \\
\hline
\end{tabular}

ACDF, anterior cervical discectomy and fusion.

$p<0.05$ (statistical significance). 
OPLL and OPLL myelopathy. For non-OPLL cases (Table 1), there was a significant correlation between the number of levels of disease to be treated and the surgical method. There was no relationship with surgical experience (Fig. 2). However, surgeons with 11 to 20 years of experience tended to favor ACDF as their primary surgical approach (Table 2). Surgeons generally preferred anterior approaches, namely ACDF (93.4\%) for one- or two-level disease $(p<0.001)$, while posterior approaches, especially laminoplasty, were far more prevalent for three- and fourlevel disease $(p<0.001)$ (Table 3). Posterior laminectomy (with or without fusion) was a rare option for surgeons. None of the participants performed disc replacement for cervical myelopathy. For ACDF, most utilized a cage with plating (40.8\%), followed by graft and plating (33.8\%), graft only (16.9\%), and cage only (8.5\%). Most surgeons preferred single-door laminoplasty for their posterior approach, using miniplates to hold the hinge open (59.2\%), followed by double-door laminoplasty (22.4\%) and singledoor laminoplasty with suture fixation (20.4\%).

For OPLL myelopathy (Table 4), anterior removal $(43.5 \%)$ or floating surgery was reserved for one-level $(p<0.001)$ disease, whereas posterior laminoplasty or laminectomy with fusion was preferred for two- to fourlevel disease ( $p=0.003$ ). More surgeons (43 versus 21 ) considered laminectomy and fusion for OPLL as compared with non-OPLL cases. There was no obvious relationship between surgical experience and the choice of surgical approach for these cases (Fig. 3). Most surgeons, regardless of experience, tended to utilize posterior approaches, either laminoplasty or laminectomy with fusion, for OPLL cases involving more than two levels (Table 5). For anterior surgery, $16.9 \%$ elected graft only, $8.5 \%$ cage only, 33.8\% graft and plating, and $40.8 \%$ cage and plating. For laminoplasty, most surgeons performed single-door

Table 3. Grouping of surgical approaches based on level of disease

\begin{tabular}{lcccc} 
& \multicolumn{3}{c}{ Surgical approach } & \\
Level of & disease & Anterior & Posterior & $\begin{array}{c}\text { Anterior and } \\
\text { posterior }\end{array}$ \\
\cline { 2 - 4 } 1 & 69 & 4 & 3 & $<0.001^{*}$ \\
2 & 58 & 12 & 5 & \\
3 & 17 & 52 & 9 & \\
4 & 4 & 64 & 3 &
\end{tabular}

laminoplasty with miniplates (59.2\%), followed by singledoor laminoplasty with sutures (20.4\%) and double-door laminoplasty (22.4\%). For posterior fusion, lateral mass

Table 4. Surgical approach for OPLL in relation to level of disease

\begin{tabular}{|cccc} 
& \multicolumn{3}{c}{ Surgical approach } \\
\cline { 2 - 3 } Levels of disease & Yes & No & $p$-value \\
\cline { 2 - 3 } & & & $<0.001^{*}$ \\
\hline Anterior removal & 21 & 58 & \\
\hline 1 & 2 & 77 & \\
\hline 2 & 0 & 79 & \\
\hline 3 & 0 & 79 & \\
\hline 4 & & & $<0.001^{*}$ \\
\hline Anterior floating of OPLL & 14 & 65 & \\
\hline 1 & 16 & 63 & \\
\hline 2 & 1 & 78 & \\
\hline 3 & 1 & 78 & \\
\hline 4 & & & 0.807 \\
\hline
\end{tabular}

Posterior laminectomy

0.807

$\begin{array}{lll}1 & 4 & 75 \\ 2 & 6 & 73 \\ 3 & 7 & 72 \\ 4 & 5 & 74\end{array}$

Posterior laminectomy and fusion

$\begin{array}{lrl}1 & 6 & 73 \\ 2 & 12 & 67 \\ 3 & 11 & 68 \\ 4 & 14 & 65\end{array}$

Posterior laminoplasty

$0.036^{*}$

\begin{tabular}{cccc}
1 & 13 & 66 & \\
\hline 2 & 19 & 60 & \\
3 & 28 & 51 & \\
\hline 4 & 25 & 54 & \\
\hline Posterior laminoplasty and fusion & & & \\
\hline 1 & 0 & 79 & \\
\hline 2 & 1 & 78 & \\
\hline 3 & 5 & 74 & \\
\hline 4 & 5 & 74 & \\
\hline Combined anterior and posterior & & & \\
\hline 1 & 0 & 79 & \\
\hline 2 & 0 & 79 \\
\hline 3 & 2 & 77 \\
\hline 4 & 3 & 76 \\
\hline
\end{tabular}

OPLL, ossification of posterior longitudinal ligament. $p<0.05$ (statistical significance). 


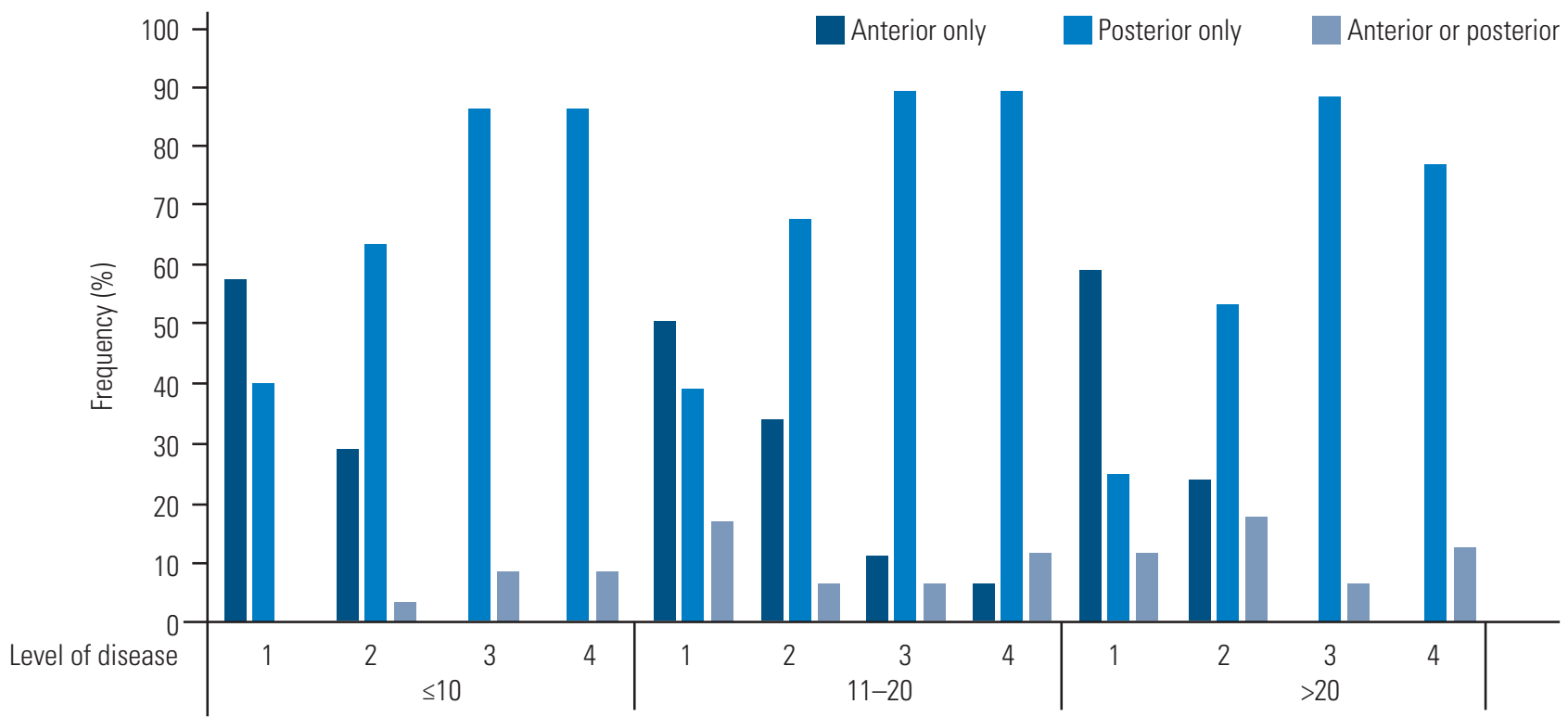

Spine surgery experience (yr)

Fig. 3. Years of surgeon's experience in relation to choice of surgical approach for cervical myelopathy caused by ossification of the posterior longitudinal ligament.

Table 5. Surgical approach for ossification of posterior longitudinal ligament based on surgical experience

\begin{tabular}{|c|c|c|c|}
\hline Years of practicing spine surgery & Disease extent/levels of disease & Surgical approach & $p$-value \\
\hline \multirow[t]{7}{*}{$\leq 10$} & 1 to 4 levels & Anterior removal & - \\
\hline & & Anterior floating & 0.545 \\
\hline & & Posterior laminectomy & 0.123 \\
\hline & & Posterior laminectomy and fusion & $0.022^{*}$ \\
\hline & & Posterior laminoplasty & $0.005^{*}$ \\
\hline & & Posterior laminoplasty and fusion & 0.063 \\
\hline & & No preference for anterior or posterior & $0.003^{*}$ \\
\hline \multirow[t]{7}{*}{$11-20$} & 1 to 4 levels & Anterior removal & - \\
\hline & & Anterior floating & 0.581 \\
\hline & & Posterior laminectomy & 0.564 \\
\hline & & Posterior laminectomy and fusion & 0.859 \\
\hline & & Posterior laminoplasty & 0.427 \\
\hline & & Posterior laminoplasty and fusion & 0.822 \\
\hline & & No preference for anterior or posterior & $0.043^{*}$ \\
\hline \multirow[t]{7}{*}{$>20$} & 1 to 4 levels & Anterior removal & - \\
\hline & & Anterior floating & 0.928 \\
\hline & & Posterior laminectomy & 0.564 \\
\hline & & Posterior laminectomy and fusion & $0.002^{*}$ \\
\hline & & Posterior laminoplasty & $0.020^{*}$ \\
\hline & & Posterior laminoplasty and fusion & - \\
\hline & & No preference for anterior or posterior & 0.210 \\
\hline
\end{tabular}

No reports of anterior removal for more than 1-level disease, none of the $>20$ years of experience surgeons selected posterior laminoplasty and fusion.

" $p<0.05$ (statistical significance). 
fixation was preferred (86.5\%).

For atlantoaxial instrumentation, C1 lateral mass screws and C2 pedicle screws were rated as most favorable $(64.6 \%)$ among the surgeons. Up to $46.9 \%$ reported $\mathrm{C} 1 / 2$ transarticular screws as their second most favorable instrumentation method, followed by $\mathrm{C} 1$ lateral mass screws and C2 laminar screws (52.0\%) and wiring (63.2\%). However, up to $51.9 \%$ reported wiring methods as their least favorable technique.

Most surgeons preferred to use the rigid neck collar for ACDF (59.4\%), corpectomy (62.3\%), corpectomy with plating $(57.1 \%)$, laminectomy $(50.0 \%)$, laminectomy with fusion (58.8\%), laminoplasty (47.6\%), C1/2 fusion (65.7\%), and occipital-cervical fusion (61.9\%). If plating was used with ACDF, the soft neck collar was most often prescribed (52.1\%). Halo immobilization was utilized in $20.6 \%$ of occipital-cervical fusions. Only $12.7 \%$ and $12.3 \%$ of surgeons allowed free mobilization after laminoplasty and ACDF with plating, respectively $(p<0.001)$. Most surgeons prescribed 6 weeks of rigid collar immobilization after surgery, except for ACDF with plating, for which 3 weeks was most commonly prescribed $(p<0.001)$.

\section{Discussion}

Being able to generalize the clinical approaches of a heterogeneous population helps surgeons to provide the best or most recommended practice. Identifying similar strategies in this highly diverse region delivers a consensus-like recommendation from a collection of 16 nations. Hence, this study imparts expert evidence from a large and assorted group of surgeons from the Asia-Pacific region. Relatively good consensus was achieved for clinical diagnosis, methods of investigation, and surgical treatment.

Despite the lack of evidence to support a particular sign or symptom for predicting outcome after treatment, the symptoms and signs necessary for the diagnosis of cervical myelopathy are quite consistent throughout the region. Previous interviews with international spine surgeons suggest that impaired gait, clumsy hands, muscle weakness, intrinsic muscle atrophy, broad-based unstable gait, and motor deficits with corticospinal distribution are the main symptoms and signs for predicting outcomes [10]. Apart from muscle weakness and motor deficits, the key clinical features reported include dexterity problems with hand function, hyperreflexia, and unsteady gait. All of these features indicate an upper motor neuron lesion, which consistently appears to be the most important to surgeons, regardless of their background or surgical experience. Similarly, nearly all surgeons utilized MRI for diagnosis and prognosis [11], and JOA score for assessment of disease severity. In addition to measuring disease severity, outcome scores are crucial for monitoring recovery after treatment. Given the strong influence of Japan in the management of cervical myelopathy in this region, it is not unexpected that most surgeons will utilize outcome assessments developed here [12-15]. Furthermore, there are some cultural similarities between several Asian countries, thereby increasing the applicability of the JOA score in this region as compared with Western countries. The JOA score has since become an important indicator of surgical treatment because of its usefulness for prognostication [16].

Some aspects of the treatment of cervical myelopathy are still controversial due to lack of solid evidence supporting one treatment over another. This is particularly true for more controversial issues such as surgery on asymptomatic and silent cervical spinal stenosis, which, although it warrants serious consideration because of its severe sequelae with minor trauma, has yet to gain significant popularity [6,17]. This is due to the unknown denominator of patients with cervical stenosis who do not experience any events. Given the higher prevalence of OPLL in the Asian continent [3], some surgeons appear to be more liberal with 'prophylactic' decompression in patients with silent cervical spinal canal stenosis. Based on knowledge of the natural history of OPLL with progressive enlargement [18], early intervention may help prevent significant sequelae, such as rapidly deteriorating cervical myelopathy or acute spinal cord injuries.

Despite some studies suggesting that anterior surgery reduces functional recovery (2.5 versus 3.6 points in the JOA score) at 1-year follow-up [19], surgical decisions are still individualized, as the overall outcomes of anterior and posterior surgery remain similar with regard to effectiveness and safety [20]. However, there are some indications for one or the other of these approaches that are well highlighted in this study. The decision for an anterior or posterior approach relies heavily on the level of disease involvement. Regardless of whether or not OPLL is present, anterior approaches are much more popular for one-level disease. ACDF and anterior removal of OPLL are most commonly utilized. The preferences for fusion options are highly variable, with equal preference for the graft or 
cage and the plate fixation methods. Without significant variability between countries or surgeon experience, it appears that the indications for anterior surgery are representative of the entire region.

Most surgeons elected posterior approaches for multilevel stenosis, with a preponderance for laminoplasty (motion-preservation) as the option of choice. For OPLL, more surgeons elected to perform posterior laminectomy and fusion to halt OPLL growth [21]. Because of the commonly observed development of narrowing in Asian cervical spines [22,23], enlargement of the spinal canal at multiple segments may provide better outcomes according to postoperative JOA scores and neurofunctional recovery rates [24]. Posterior indirect decompression may also be safer for multilevel OPLL involvement [25]. Most surgeons selected miniplates for fixation in laminoplasty. Variations in fixation methods due to cost concerns have been reported, but use of these rigid devices was the preferred option for most surgeons.

The use of postoperative immobilization was highly variable among the participants. About half the surgeons utilized rigid neck collars for any surgical procedure. In anterior surgery, and particularly in fusion operations, the use of collars had no effect on clinical outcomes or complication rates [26]. However, early use of collars may improve axial neck pain in laminoplasty [5]. Most surgeons suggest 6 weeks of collar use only to protect their fixation or fusion procedures. The duration of use is relatively short, and prolonged use may cause more postoperative axial neck pain [27-29].

Surgeons from three countries in the Asia-Pacific region did not participate in this study. However, most of the highly populated countries, including China, India, Japan, and Korea, were included, and thus the results should well represent this vastly diverse region. This study provides a general overview of various aspects of cervical myelopathy treatment, but further investigation into more intricate details of the surgical approaches should be performed. These may include the practice of minimally invasive surgery, routine decompression lateral to the uncovertebral joints during anterior discectomy, and methods of neuromonitoring.

\section{Conclusions}

The Asia-Pacific region comprises many countries with different ethnic and cultural backgrounds. Providing an overview of expert opinions in this region helps this large, diverse group of surgeons streamline their management strategies to fit the common norm or generally accepted practice. This study provides the first comprehensive assessment of Asia-Pacific surgeons' view of cervical myelopathy treatment, and from our results, several treatment recommendations have been generated. Cervical myelopathy is commonly diagnosed by the presence of finger dexterity problems, hyperreflexia, and gait disturbances. Prophylactic or early decompression surgery should be considered for patients with silent cervical spinal stenosis, particularly in the presence of OPLL. In such cases, posterior canal-widening surgery by means of laminoplasty or laminectomy with fusion is preferred. Anterior decompression is suggested primarily for onelevel OPLL disease.

\section{Conflict of Interest}

No potential conflict of interest relevant to this article was reported.

\section{Author Contributions}

All authors contributed to the study design, interpretation, drafting and final approval of the work.

\section{References}

1. Kalsi-Ryan S, Karadimas SK, Fehlings MG. Cervical spondylotic myelopathy: the clinical phenomenon and the current pathobiology of an increasingly prevalent and devastating disorder. Neuroscientist 2013;19:409-21.

2. Smith ZA, Buchanan CC, Raphael D, Khoo LT. Ossification of the posterior longitudinal ligament: pathogenesis, management, and current surgical approaches: a review. Neurosurg Focus 2011;30:E10.

3. Fehlings MG, Ibrahim A, Tetreault L, et al. A global perspective on the outcomes of surgical decompression in patients with cervical spondylotic myelopathy: results from the prospective multicenter AOSpine international study on 479 patients. Spine (Phila Pa 1976) 2015;40:1322-8.

4. Cheung JPY, Cheung PWH, Cheung AYL, Lui D, Cheung KMC. Comparable clinical and radiological outcomes between skipped-level and all-level 
plating for open-door laminoplasty. Eur Spine J 2018;27:1365-74.

5. Cheung JPY, Cheung PWH, Law K, et al. Postoperative rigid cervical collar leads to less axial neck pain in the early stage after open-door laminoplasty-a single-blinded randomized controlled trial. Neurosurgery 2018 Aug 3 [Epub]. https://doi.org/10.1093/ neuros/nyy359.

6. Shigematsu H, Cheung JP, Mak KC, Bruzzone M, Luk KD. Cervical spinal canal stenosis first presenting after spinal cord injury due to minor trauma: an insight into the value of preventive decompression. J Orthop Sci 2017;22:22-6.

7. Williams R, Cheung JP, Goss B, et al. An international multicenter study assessing the role of ethnicity on variation of lumbar facet joint orientation and the occurrence of degenerative spondylolisthesis in Asia Pacific: a study from the AOSpine Asia Pacific Research Collaboration Consortium. Global Spine J 2016;6:35-45.

8. Samartzis D, Cheung JP, Rajasekaran S, et al. Is lumbar facet joint tropism developmental or secondary to degeneration?: an international, large-scale multicenter study by the AOSpine Asia Pacific Research Collaboration Consortium. Scoliosis Spinal Disord 2016;11:9.

9. Samartzis D, Cheung JP, Rajasekaran S, et al. Critical values of facet joint angulation and tropism in the development of lumbar degenerative spondylolisthesis: an international, large-scale multicenter study by the AOSpine Asia Pacific Research Collaboration Consortium. Global Spine J 2016;6:414-21.

10. Techy F, Benzel EC. Predictors of outcome in patients with cervical spondylotic myelopathy undergoing surgical treatment: the evidence and the international common practice. World Neurosurg 2014;81:503-7.

11. Nouri A, Martin AR, Nater A, et al. Influence of magnetic resonance imaging features on surgical decision-making in degenerative cervical myelopathy: results from a global survey of AOSpine international members. World Neurosurg 2017;105:864-74.

12. Cheung PW, Wong CK, Lau ST, Cheung JP. Psychometric validation of the adapted traditional Chinese (Hong Kong) version of the Japanese Orthopaedic Association Cervical Myelopathy Evaluation Questionnaire (JOACMEQ). Spine (Phila Pa 1976) 2018;43:E242-9.
13. Fukui M, Chiba K, Kawakami M, et al. An outcome measure for patients with cervical myelopathy: Japanese Orthopaedic Association Cervical Myelopathy Evaluation Questionnaire (JOACMEQ): part 1. J Orthop Sci 2007;12:227-40.

14. Kato S, Oshima Y, Oka H, et al. Comparison of the Japanese Orthopaedic Association (JOA) score and modified JOA (mJOA) score for the assessment of cervical myelopathy: a multicenter observational study. PLoS One 2015;10:e0123022.

15. Tanaka N, Konno S, Takeshita K, et al. An outcome measure for patients with cervical myelopathy: the Japanese Orthopaedic Association Cervical Myelopathy Evaluation Questionnaire (JOACMEQ): an average score of healthy volunteers. J Orthop Sci 2014;19:33-48.

16. Machino M, Yukawa Y, Imagama S, et al. Surgical treatment assessment of cervical laminoplasty using quantitative performance evaluation in elderly patients: a prospective comparative study in 505 patients with cervical spondylotic myelopathy. Spine (Phila Pa 1976) 2016;41:757-63.

17. Aebli N, Ruegg TB, Wicki AG, Petrou N, Krebs J. Predicting the risk and severity of acute spinal cord injury after a minor trauma to the cervical spine. Spine J 2013;13:597-604.

18. Katsumi K, Watanabe K, Izumi T, et al. Natural history of the ossification of cervical posterior longitudinal ligament: a three dimensional analysis. Int Orthop 2018;42:835-42.

19. Fehlings MG, Barry S, Kopjar B, et al. Anterior versus posterior surgical approaches to treat cervical spondylotic myelopathy: outcomes of the prospective multicenter AOSpine North America CSM study in 264 patients. Spine (Phila Pa 1976) 2013;38:2247-52.

20. Lawrence BD, Jacobs WB, Norvell DC, Hermsmeyer JT, Chapman JR, Brodke DS. Anterior versus posterior approach for treatment of cervical spondylotic myelopathy: a systematic review. Spine (Phila $\mathrm{Pa}$ 1976) 2013;38(22 Suppl 1):S173-82.

21. Lee JJ, Shin DA, Yi S, et al. Effect of posterior instrumented fusion on three-dimensional volumetric growth of cervical ossification of the posterior longitudinal ligament: a multiple regression analysis. Spine J 2018 Mar 8 [Epub]. https://doi.org/10.1016/ j.spinee.2018.03.002.

22. Koyama T, Handa J. Cervical laminoplasty using apa- 
tite beads as implants: experiences in 31 patients with compressive myelopathy due to developmental canal stenosis. Surg Neurol 1985;24:663-7.

23. Shigematsu H, Ueda Y, Koizumi M, et al. Does developmental canal stenosis influence surgical results of bilateral open-door laminoplasty for cervical spondylotic myelopathy? J Neurosurg Spine 2008;9:35862.

24. Qin R, Chen X, Zhou P, Li M, Hao J, Zhang F. Anterior cervical corpectomy and fusion versus posterior laminoplasty for the treatment of oppressive myelopathy owing to cervical ossification of posterior longitudinal ligament: a meta-analysis. Eur Spine J 2018;27:1375-87.

25. Nayak NR, Piazza M, Milby A, et al. Surgical approaches for the treatment of multilevel cervical ossification of the posterior longitudinal ligament: results of a decision analysis. World Neurosurg 2018;112:e375-84.

26. Campbell MJ, Carreon LY, Traynelis V, Anderson PA. Use of cervical collar after single-level anterior cervical fusion with plate: is it necessary? Spine (Phila $\mathrm{Pa}$ 1976) 2009;34:43-8.

27. Kawaguchi Y, Kanamori M, Ishiara H, Nobukiyo M, Seki S, Kimura T. Preventive measures for axial symptoms following cervical laminoplasty. J Spinal Disord Tech 2003;16:497-501.

28. Kowatari K, Ueyama K, Sannohe A, Yamasaki Y. Preserving the $\mathrm{C} 7$ spinous process with its muscles attached: effect on axial symptoms after cervical laminoplasty. J Orthop Sci 2009;14:279-84.

29. Shiraishi T, Fukuda K, Yato Y, Nakamura M, Ikegami T. Results of skip laminectomy-minimum 2-year follow-up study compared with open-door laminoplasty. Spine (Phila Pa 1976) 2003;28:2667-72. 